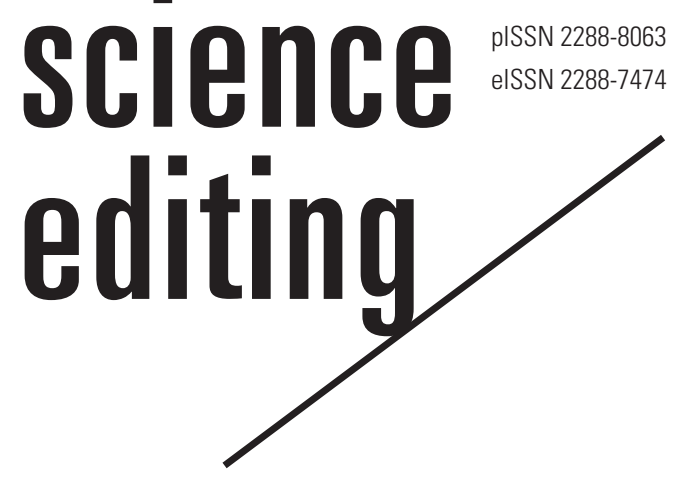

\title{
How Asian publishers can compete with publishers in Europe and North America
}

\author{
Sun Huh \\ ALPSP 10th Anniversary Conference and Awards \\ Date: September 13-15, 2017 \\ Venue: Grand Hotel Huis ter Duin, Noordwijk, Netherlands \\ URL address: https://www.alpsp.org/2017-Programme
}

Department of Parasitology and Institute of Medical Education, College of Medicine, Hallym University, Chuncheon, Korea

This was the second time that I attended the Association of Learned and Professional Society Publishers (ALPSP, https://www.alpsp.org) conference and awards. I attended the eighth conference in London in 2015. The ALPSP is the one of the largest organizations of scholarly publishers, with offices located in Hertfordshire, United Kingdom. In North America, the corresponding organization is the Society for Scholarly Publishing (https://www.sspnet.org/). I was frequently asked whether I was a publisher or an editor. I am the editor of Journal of Educational Evaluation for Health Professions, but I have also worked as a managing editor. In the context of this conference, I was considered to be an editor-publisher. It was difficult to find other editor-publishers at the conference; in fact, I only met 1 Chinese editor-publisher of a physics journal at the editorial board meeting of Learned Publishing, the official journal of the ALPSP. This conference was originally a meeting of publishers, not editors. Most members whom I met were publishers who were employed by publishing companies.

The purpose of my visit to this conference was as follows: first, I planned to attend the editorial board meeting of Learned Publishing. I was invited to be an associate editor in 2015 by the editor, Pippa Smart. Second, I hoped to meet publishers and to interact with them to learn about their perspectives and activities in more detail; third, I intended to find out about recent innovations by publishers in order to catch up with recent progress in the scholarly publishing.

The Grand Hotel Huis ter Duin had a beachfront location on the Atlantic. On the first day, the first keynote address, "Sloppy science, selective reporting and the replication crisis," was presented by Lex M Bouter of Brije University, Amsterdam. I remembered his speech from the

Received: January 31, 2018 Accepted: February 2, 2018

Correspondence to Sun Huh shuh@hallym.ac.kr

ORCID

Sun Huh

http://orcid.org/0000-0002-8559-8640 plenary lecture he gave at the 13th European Association of Science Editors Conference held on June 10 to 12, 2016, in Strasbourg, France [1]. Because I had heard the content before, I was able to understand the presentation more thoroughly. He said that although sloppy science is not categorized as research misconduct, publishing scientifically flawed articles is a waste of journal space. Furthermore, it may cause bias in meta-analyses. He stressed that editors should be cautious about sloppy science and should prevent it. He suggested open access to ensure the 
transparency of reports and open data policies to ensure the transparency of data. He also suggested the following approach to promote transparency: not to focus on the impact factor and citations, but to reward proper protocols, studies reporting negative data, data sharing, replication, dissemination, and application. Transparency can be achieved through nudging and forcing by the institutional review board, funders, and journals by reviewing study protocols at the first stage of research. If the study protocol is approved, the researchers can conduct the study and obtain the data. The journal can also review the study protocol as a first step, and then the data and interpretation can be submitted after data collection. Funders also can pay step by step. The feasibility of journals engaging in nudging and forcing via step-by-step procedures should be considered more carefully. In such a system, researchers would submit each study protocol to the institutional review board and to a journal. After receiving approval, they would conduct the research and submit the final data. Therefore, this system would involve 2-step submissions. I am not sure whether I would be able to adopt this system for my journal.

My journal was the first mover in Korea to adopt an open data policy, starting in March 2016 [2]. This policy was smoothly established, without any difficulties, thanks to the cooperation of authors [3]. An open data policy may be able to guarantee replicability. However, if the raw data themselves are falsified, it would be difficult to check the scientific validity of a study. Although no scientists are expected to provide falsified raw data, there was a report that pooled weighted average of $1.97 \%(n=7 ; 95 \%$ confidence interval, 0.86 to 4.45$)$ of scientists admitted to have fabricated, falsified or modified data or results at least once-a serious form of misconduct by any standard [4]. Other presentations and discussions are available from: https://www.alpsp.org/2017-Programme and YouTube (https://www.youtube.com/playlist?list = PLYB4k71c VIeXa2ddgXqIJiCi2MmX4Z39_) [cited January 30, 2018]. One of the most interesting topics was the Escalex project, by IFIS Publishing, which was 1 of the 7 finalists for the ALPSP Awards for Innovation in Publishing. This project provides food regulatory information from various countries. A variety of information technologies were utilized to build this system. The award in 2017 was presented to the Publons and Source Data projects from EMBO. Publons was founded in 2013, and is a pioneering global peer-review data and recognition platform. It was a breakthrough idea. The Source Data project provides dataset and metadata linking, data searching, and dissemination of data. Data have become more important year by year. EMBO's approach to data was beautiful and intuitive.

As I listened to the speeches at the conference, I noticed that there were no presentations by publishers from Asia, as no Asian publishers were invited to present at the conference. This may have been because there are not many international publishing companies in Asia and because publishers from Asia could attend the ALPSP conference on an individual basis. I met a publisher from Profeza, India (http://profeza. $\mathrm{com} /$ ) in the lobby. He explained their system for open data and laboratory data and how they were linked to in the articles. It was fantastic to see a direct and simple link from articles to data. It may be convenient for readers and researchers to read the article and check the data at the same time.

At lunch time on the second day, there was an editorial board meeting of Learned Publishing led by Pippa Smart, the editor of the journal. I made the following suggestions that have already been introduced at my journal: first, adopting an open data policy; second, Medline indexing; third, including a declaration of conflicts of interest; and fourth, requesting informed consent agreements for articles with human subjects. My suggestions were all discussed and accepted, and the follow-up work was started. I am especially interested in Medline indexing, which may provide more convenient searching and better visibility. I was very happy that my suggestions were accepted and processed after the board meeting. Other topics were also discussed, including Altmetrics and marketing. A new leaflet was introduced and the new name card of the journal was provided to the editorial board members. Although the board meeting took approximately an hour, the discussion was very active. It was particularly impressive that we were connected with board members who could not attend the conference via telephone or Internet. I could hear the voices of fellow board members through a speaker. I also hope to adopt this tele-meeting system for my journal's editorial

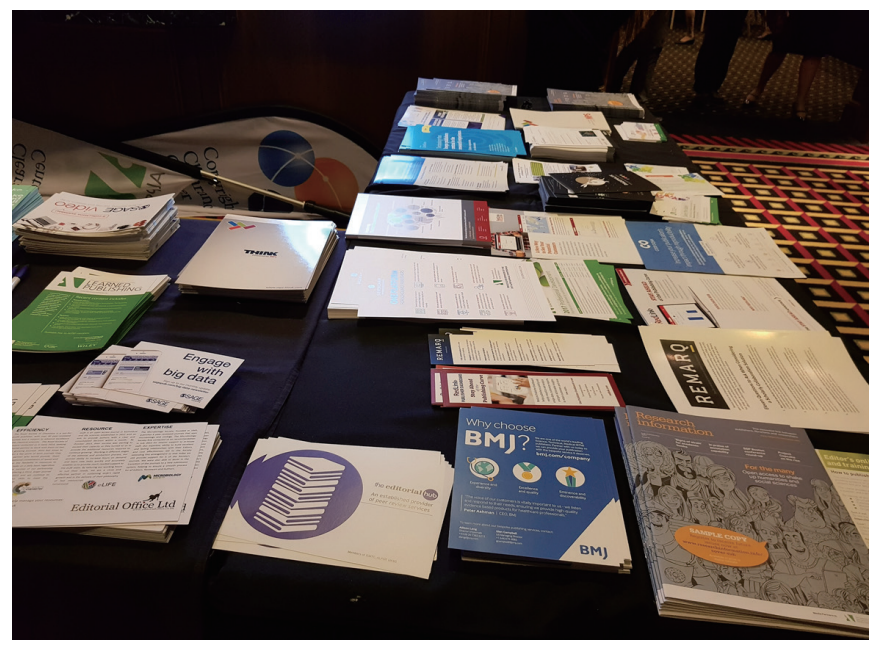

Fig. 1. Pamphlets displayed at the lobby of the 10th Association of Learned and Professional Society Publishers conference and award. 
board meetings, because my journal's board members are from 36 countries.

I enjoyed talking with attendees in the lobby and dining room. I asked publishers whether they had adopted Journal Article Tag Suite (JATS) extensible markup language (XML) for full-text article XML. Staff members from both Springer and Wiley told me that they generally used their own XML for full-text articles, although they produced JATS XML for deposit to PubMed Central. In Korea, all academic societies have adopted JATS XML for full-text articles. Large international publishers may have their own systems for their unique databases. The wide range of pamphlets displayed in the lobby was another source of information (Fig. 1). Many publishers would like to raise awareness of their products or technologies by providing pamphlets. I collected many pamphlets and have frequently read them after returning home.

After dinner on the second day, there was a quiz hour. The attendees at each table formed a team, answering questions on science, music, novels, and other topics. After calculating the total score of each team, the winning team was selected. My team was the winner, so I also received a small prize. This was a pleasant form of entertainment, as attendees relaxed and cooperated with each other to find the answers.

It was hard for me to travel from East Asia to Europe. The time lag was 8 hours. However, I was able to enjoy the conference and to meet colleagues. Furthermore, after the conference I visited Leiden University and enjoyed the beautiful botanical museum there. Before returning to Korea, I attended the season opening opera concert in Amsterdam. Enjoying the culture while attending a conference abroad was another source of joy.

What lessons can Asian editors, including myself, learn from attending the ALPSP conference? First, Asian editors should strive to promote Asia-based international publishing companies to the international level so that they can be competitive with Europe or North America-based international publishing companies. Most scholarly journals from Asia are published by academic societies or non-profit organizations or institutes; therefore, they have no stable income model. Publishers have provided the cost of publication. I suggest establishing a non-profit cooperative of a number of academic societies jointly with printing, manuscript editing, engineering, English proofreading, and illustration companies. Therefore, the cooperative can train professionals in editing and publishing. Second, associations of science editors in Asia, such as the Council of Asian Science Editors, should play the role of information providers to Asian editors by delivering journals and newsletters and conducting regular meetings for training. The journal market has evolved rapidly in recent years, so we, as Asian editors, should catch up with this pace of development as soon as possible.

\section{Conflict of Interest}

No potential conflict of interest relevant to this article was reported.

\section{References}

1. Huh S. What can Asian editors contribute to European editors? Sci Ed 2016;3:122-4. https://doi.org/10.6087/kcse.79

2. Huh S. Promotion to MEDLINE, indexing with Medical Subject Headings, and open data policy for the Journal of Educational Evaluation for Health Professions. J Educ Eval Health Prof 2016;13:14. https://doi.org/10.3352/jeehp. 2016.13.14

3. Huh S. Establishment of an open data policy for Journal of Educational Evaluation for Health Professions, appreciation for invited reviewers, and acknowledgement of volunteers who made audio recordings. J Educ Eval Health Prof 2017;14:37. https://doi.org/10.3352/jeehp.2017.14.37

4. Fanelli D. How many scientists fabricate and falsify research? A systematic review and meta-analysis of survey data. PLoS One 2009;4:e5738. https://doi.org/10.1371/ journal.pone. 0005738 